\title{
Between the crystalline and the chaotic
}

Dave Everitt, Institute of Creative Technologies (IOCT),

De Montfort University, Leicester, UK.

Published in Digital Creativity, 2013, Vol. 24, No. 3, 1 - 5, http://dx.doi.org/10.1080/14626268.2013.813379 (forthcoming), Taylor \&

Francis

\section{Abstract}

This short feature documents elements of research in advance of a long-term work. Rather than a technical account or retrospective, the aim is to demonstrate by example how research itself is a primary process, illustrated by work carried out at the IOCT during the last few years. When creative output is appraised only from its visible results, something is lost in the telling; carefully selected public facets rarely convey the depths of the underlying lines of inquiry. The research covered is for a work that generates quantitative data, driving visualisations of human movement, proximity and orientation. The aim is to reveal how this literal data reflects our relationships to one another in social settings. To contextualise the current process, complex systems, dynamic pattern formation and other works are also mentioned. As a personal account, it seemed appropriate to supplement the usual academic passive voice with first-person narrative.

\section{Keywords}

process, sketch, complexity, social behaviour, pattern

\section{The hidden iceberg of process}

The truism is that ideas are cheap, while successful execution constitutes the greater percentage of the necessary work. Yet in creative activities, the visible part of the crystallised 'iceberg' of concrete outcomes exposed to public view is supported by a body of 'execution' of far greater dimensions and with less immediately apparent structure. The visible exists only because of the density of the underlying research with its encrusted strata, fractures and density. The final solidity of a work can be the product of false starts, detours, experiments, broken collaborations and funding applications. Producing readable outcomes from such a disparate and frequently gruelling journey requires a parallel series of activities that captures smaller particles of value along the way, crystallising them into tangible forms that express their value. To appreciate complex longterm works, research therefore needs to be as visible as finished output.

As well as preparing and exhibiting another long-term work (Everitt 2011a), my practice over the last few years has been largely research-based. After some years of project-based focus, around 2011 I began doodling with intent, both digitally and on paper (Everitt 2011b). Some sketches are technical experiments that have contributed in unforeseen ways to other work; for example Gimme 5 (Everitt 2010) exploits the unpredictable tags people associate with images, and the consequent leaps of meaning created by the combination of literal computer search processing and imaginative human curiosity; this sketch led to research for a more complex online work in progress. 
Sketches thereby pave the way for-and even determine-future direction. The research in Figure 1 concerns the simulation of multiple human movements over time.
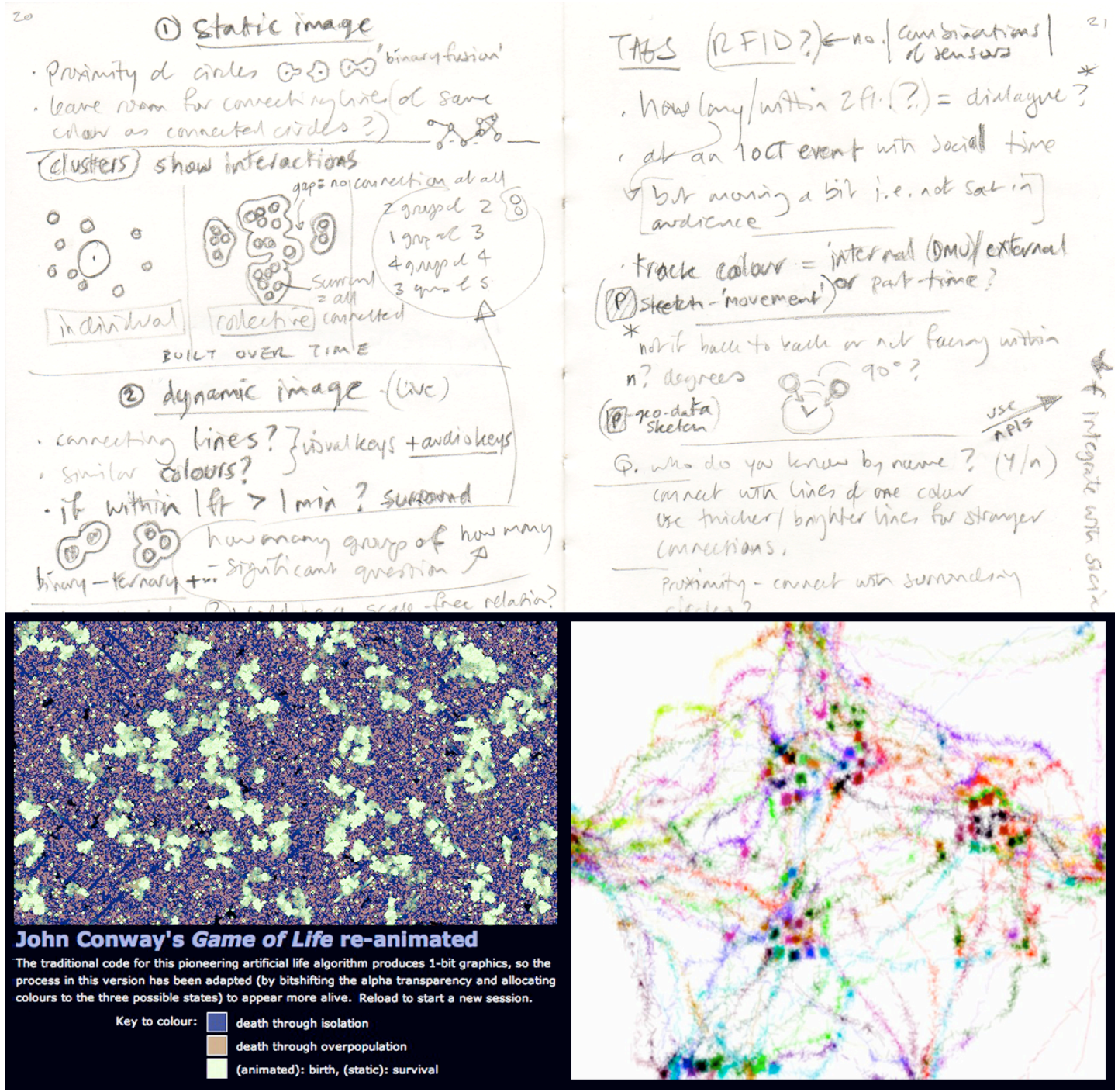

Figure 1: Two manual sketches and two examples of resulting digital sketches.

After pencil notes (Figure 1, top) the first step was to adapt a Processing sketch (processing.org) of the seminal 'game of life' (Callahan, n.d.). I especially wanted to animate the complex activity of the three simple rules as they play out, generating 'living' cells across the terrain (Figure 1, bottom left). A similarlybased method known as agent-based modelling or 'ABM' (Axelrod and Tesfatsion 2007 ) is used to map issues such as social segregation (Schelling 1978) and interaction (Klemm et al 2003); much of my background research has drawn on this field. As a response to these computer simulations, the project proposal argues the case for an artwork supported by a system accumulating data about actual human motion, with the aim of revealing patterns of relational activity-a kind of 'reversed ABM' in that the visuals are driven by real, not simulated, human data. The research question is whether this will give visual form to our relationships in social spaces by revealing patterns concealed within human movement data. 
To test possible visualisations of motion tracks from the paths of separate individuals in a given space, I coded a Processing sketch (Figure 1, bottom right) with a 'hand-drawn' algorithm that draws lines of moderate opacity, rapidly at random angles with different colours for each track, the tracks in this case simulated by mouse movement. The opacity and sketchiness of the lines produces a greater density of colour where speed is slowest e.g. when someone stands still. The illustration highlights how people cluster, pause, pair up, or remain in close proximity to other individuals and groups over time.

The next stage of the work was a collaboration with former IOCT researcher Gareth Howell. Forty-six GPS-tracked walks around the UK city of Nottingham provided real-world quantitative data for test visualisations (Figure 2). Use of GPS data is fairly widespread in commercial applications and served well for this test, but the long-term aim is to capture data from collective human motion and proximity, particularly at indoor locations and at a higher resolution, then visualise it to investigate any patterns. This is a different kind of problem than both the rule-based programmatic modelling of ABM and the tracking of motion using velocity and location vectors ('the most basic building block for programming motion' [Shiffman 2012]). Current GPS resolution is too coarsegrained; besides, other data such as orientation is required to determine where individuals are facing.

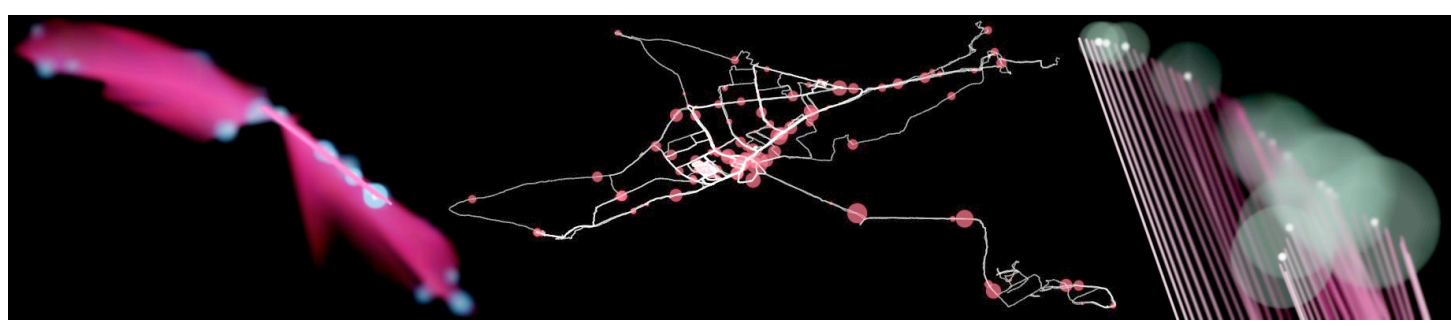

Figure 2. Test visualisations of motion tracks using GPS data

The central image in Figure 2 shows the literal tracking of forty-six walks along actual streets, with circles that mark distances travelled. The two images either side are from happy accidents in the coding of one of the vector coordinates. Using the same data as the central image, those accidental visualisations are less literal, yet still informative representations of the forty-six walks and distances covered; each journey fading over time as successive files are read in and displayed.

\section{Sketches iterated are process made visible}

Although some of these small works run consistently and appear engaging enough to be presented at a larger public scale as finished work, it would be dishonest to present them as such-as sketches they:

1. solve a single issue or try a particular approach, visualisation or data set;

2. contain inflexible hard-coded values and unrefined code untested for robust long-running public engagement;

3. are un-optimised, use processing power inefficiently, and are therefore only suitable as proof of concept;

4. are stepping-stones towards a more complete output. 
However incomplete, each sketch is part of the 'wandering walk' towards future public works. Because of the time-consuming demands of digital work, making facets of the process explicitly visible at various stages is especially important during the period this project will take to reach the stage of an initial interactive public work. Sharing sketches at this stage is an attempt to make the long-term research more transparent, especially when drawing on multiple disciplines. Documenting this journey is where the real narrative resides. While it may be true that periodic outputs are crystallised for public access at the expense of their evolving dynamism, making them available periodically creates a holistic picture of the 'work behind the work'. This approach relates to tenets made popular by the agile software movement (Beck et al 2001): build working examples people can use, get feedback, improve and repeat.

Using code as a creative tool requires continual professional development; one hard-learned lesson is the need for version control (Chacon 2009). 'Rolling back' to a previous working version has saved my sanity when things go awry for no detectable reason except-perhaps-a tired brain. The hidden advantage of version control is that every stage of a work carries within its archive all previous iterations, like an evolutionary tree. For works that become historical artefacts, version control enables the journey to be captured in the process.

\section{Human cognition and the appreciation of pattern}

Stepping back to consider the broader context behind this work on human movement, I've become aware of parallels between social activity, the creative process, complex dynamic systems and pattern formation in the natural world (Everitt 2011c). An amateur but active interest in complexity theory and dynamic pattern formation has become a dominant theme in my current research, leading directly to the project under consideration in this paper.

This theme first appeared in cubeLife, an interactive artwork initially exhibited in 1999, with heartbeats generating sonic loops and visuals created by magic cubes (Everitt 2011a). These 3-dimensional matrices of sequential integersarranged to sum to the same number along any row, column or diagonalparadoxically produce both diverse symmetrical and chaotic patterns from a single set of rules (Heinz 2011). Magic matrices are finite and static, but complex behaviours in the natural world form patterns that transcend specific materials and span disciplines (Ball 1998). Since then, my interest in pattern has expanded towards an investigation of human social activity.

Critical appraisals assert the undesirability of extremes and the desirability of that which lies between. With terms such as 'unstructured', 'daub', 'cacophony', 'clumsy', 'ugly' ... 'formulaic', 'clichéd', 'mannered', 'rigid' ... 'elegant', 'beautiful', 'balanced', it seems that we require a kind of aesthetic 'Goldilocks zone' (see Grossman [2013] for this astronomical term in context). As with any complex behaviour, a combination of flexibility plus rules produces outcomes unpredictable enough to engage or inspire, and variable enough to sustain interest. As part of the natural world, the suggestion is that we cannot avoid embodying in our activities and interactions the very same properties that shape the natural processes of our existence; this is the deeper question behind the work towards which these sketches on human movement are contributing. 
Conversations with representatives from other disciplines have made me aware that these kinds of data is worth sharing with the wider research community, especially when combined with visualisations that might reveal patterns otherwise difficult to identify. The responsibility of designing and creating hardware and software for an interactive work that can generate such data is a significant research challenge. Documenting the research is therefore as much a part of the work as any resultant output.

\section{References}

Axelrod and Tesfatsion. 2007. "On-Line Guide for Newcomers to Agent-Based Modeling in the Social Sciences". Accessed Feb 13 2013, http://www.econ.iastate.edu/tesfatsi/abmread.htm

Ball, Philip. 1998. The Self-Made Tapestry: Pattern Formation in Nature. Oxford University Press.

Beck, Kent et al. "Manifesto for Agile Software Development". Accessed Feb 13 2013, http://agilemanifesto.org

Callahan, Paul: "What is the Game of Life?" Accessed Feb 13 2013, http://www.math.com/students/wonders/life/life.html

Chacon, Scott. 2009. "Pro Git: 1.1 Getting Started - About Version Control". Accessed Feb 13 2013, http://git-scm.com/book/en/Getting-Started-AboutVersion-Control

Everitt, Dave. 2010. “Gimme 5”. Accessed Feb 14 2013, http://daveeveritt.org/sketches/give-me-things/

Everitt, Dave. 2011a. cubeLife and cubeLife II. CubeLife first developed at Creativity and Cognition Research Studios, Loughborough University, UK and shown in 1999; cubeLife II completed at the Institute of Creative Technologies, exhibited April 2011, DMU Cube Gallery, Phoenix Square, Leicester.

Everitt, Dave. 2011b. “Selected sketches”. Accessed Feb 14 2013, http://daveeveritt.org/sketches/

Everitt, Dave. 2011c. "Is Creativity a 'natural' process?". in: Ascott, R., Girao, M., ed. Presence in the Mindfield: Art, Identity and the Technology of Transformation. Lisbon, Portugal: Artshare-Universidade de Aveiro, pp. 103-107. ISBN 978-972789-356-0

Grossman, Lisa. 2013. "Earth and others lose status as Goldilocks worlds". New Scientist: Space. Accessed Apr 18 2013, http://www.newscientist.com/article/dn23118-earth-and-others-lose-status$\underline{\text { as-goldilocks-worlds.html }}$

Heinz, Harvey. 2011. "Magic Cubes”. Accessed Feb 14 2013, http://www.magicsquares.net/magic cubes index.htm

Klemm, Konstantin et al. 2003. "Nonequilibrium transitions in complex networks: A model of social interaction”. Physical Review E 67, 026120. The 
American Physical Society. Accessed Feb 14 2013,

http://ifisc.uib.es/ victor/Cult/culturnet.pdf

Schelling. 1978. "Micromotives and Macrobehavior", New York, NY: Norton.

Shiffman, Daniel. 2012. “The Nature of Code”. Accessed Feb 14 2013, http://natureofcode.com 


\section{Relevant biography}

I have been a research fellow at the Institute of Creative Technologies, De Montfort University, Leicester since 2007, having completed projects as diverse as the 3D modelling of archaeological artefacts, and interfaces to energy monitoring systems designed to encourage the social awareness of environmental impact. Apart from early doodles during the 80s in the programming language Logo, and (with more intent) Apple's Hypercard and Hypertalk language, current engagement with technology in art practice began in 1998 with a residency at the former Gallery of the Future and, until 2002, in collaborations as a visiting researcher at Creativity and Cognition Research Studios, both at Loughborough University. Work on motion tracking carried out at that time features on the cover of 'Explorations in Art and Technology' (Candy \& Edmonds, 2002, Springer). 